\title{
SAGITTARIUS A* COMPANION S0-2: A PROBE OF VERY HIGH MASS STAR FORMATION
}

\author{
ANDREW GOULD \\ Department of Astronomy, Ohio State University, Columbus, OH 43210-1173; gould@astronomy.ohio-state.edu \\ AND \\ Alice C. Quillen \\ Department of Astronomy, University of Rochester, Rochester, NY; aquillen@pas.rochester.edu \\ Received 2003 February 20; accepted 2003 April 11
}

\begin{abstract}
The star S0-2, which is orbiting Sgr A* with a $15 \mathrm{yr}$ period, almost certainly did not form in situ. We propose that it was injected into this close orbit by the tidal disruption of a massive-star binary, whose primary was more massive than S0-2 and at least $60 M_{\odot}$. From numerical integrations we find that $1 \%-2 \%$ of incoming binaries with closest approach equal to $130 \mathrm{AU}$ leave the secondary in an orbit with eccentricity within 0.01 of that of S0-2. If additional stars are found orbiting Sgr $\mathrm{A}^{*}$ with relatively short periods, they could be used to probe the formation of massive stars in the Galactocentric region, even though the massive stars themselves have long since perished.
\end{abstract}

Subject headings: binaries: general — Galaxy: center — stars: early-type — stars: formation — stars: fundamental parameters — stars: kinematics

On-line material: color figure

\section{INTRODUCTION}

Almost a decade of high-resolution infrared observations of the Galactic center have revealed at least three stars that show substantial proper-motion acceleration due to the supermassive black hole $(\mathrm{BH})$ associated with $\mathrm{Sgr} \mathrm{A}^{*}$ (Eckart \& Genzel 1996; Ghez et al. 1998, 2000, 2003a; Eckart et al. 2002; Schodel et al. 2002). The most striking of these is $\mathrm{S} 0-2$, which has a peribothron of only $q=125 \mathrm{AU}$ and a period of only $P=15$ yr. S0-2 has a number of important potential applications. For example, by combining radial velocity and astrometric observations of $\mathrm{S} 0-2$ one could measure the mass, the distance, and the three components of motion of Sgr A* (Salim \& Gould 1999), which is believed to lie almost exactly at the Galactic center.

However, the most remarkable characteristic of the Sgr A*/S0-2 "visual binary" is that it exists at all (Ghez et al. 2003a). S0-2 cannot have formed in place: the gas cloud from which it would have condensed could never have been larger than $\sim\left(m_{1} / M_{\odot}\right)^{1 / 3}$ AU or it would have been tidally disrupted. Here $m_{1}$ is the mass of S0-2. Hence, it must have formed at a much larger distance from Sgr A* and was either originally on a highly eccentric orbit that took it within $\sim 125 \mathrm{AU}$ of Sgr A* or was perturbed onto such an orbit. Then, it would have had to have been perturbed again at peribothron to bring its eccentricity from $(1-e) \ll 1$ to $(1-e) \sim 0.13$. It is this last step that is problematic.

Spectra at $2 \mu \mathrm{m}$ taken by Ghez et al. (2003a) exhibit $\mathrm{Br} \gamma$ and $\mathrm{He} \mathrm{I}$ absorption lines and lack a $\mathrm{CO}$ band head. These features suggest that $\mathrm{S} 0-2$ is an $\mathrm{O} 8-\mathrm{B} 0$ dwarf star and so is massive, $m_{1} \sim 15 M_{\odot}$, and young, $\lesssim 10 \mathrm{Myr}$. The youth of the star puts a strong limit on the length of time available to scatter the star into its current orbit since formation. The dynamical friction time for such a star in an orbit of semimajor axis $a \sim 10^{4} \mathrm{AU}$, where it might plausibly form, would be $t_{\mathrm{df}} \sim 300 \mathrm{Myr}\left(a / 10^{4} \mathrm{AU}\right)^{-1 / 4}$, even assuming that the stellar profile continued as a power law down to very small radii, as suggested may be the case by Alexander (1999). Since several dynamical friction times would be required to achieve the orbit of S0-2, this appears impossible. ${ }^{1}$

In this paper we propose that the orbit of $\mathrm{S} 0-2$ was a result of the disruption of a massive-star binary in the tidal field of the massive black hole at the Galactic center. When the binary disrupts, one star is ejected with energy greater than its incoming energy, leaving the other star, $\mathrm{S} 0-2$, in a bound orbit with the black hole. Such "exchange reactions," in which one component of a binary is captured by a more massive star, have previously been studied in the context of globular clusters (Heggie, Hut, \& McMillan 1996). In $\S 2$, we show analytically that for this mechanism to work, the former binary companion of S0-2 must have been very massive, of order $100 M_{\odot}$. In $\S 3$, we confirm this estimate by numerical simulation, showing that the primary star must be $m_{2} \gtrsim 60 M_{\odot}$. In $\S 4$, we discuss how the detection of additional short-period $(P \lesssim 100$ yr) "low"-eccentricity $(e \lesssim 0.95)$ companions to Sgr A* could be used to probe very high mass star formation in the inner pc of the Galactic center. In Appendix A, we discuss the mechanisms by which such massive binaries might be fed to Sgr A*.

\section{ANALYTIC ESTIMATES}

The specific binding energy of S0-2 today is

$$
E_{b}=\frac{G M_{\mathrm{bh}}(1-e)}{2 q},
$$

where $e=0.87$ and $q=125 \mathrm{AU}$ are the eccentricity and peribothron of its orbit, and $M_{\mathrm{bh}}=3 \times 10^{6} M_{\odot}$ is the mass of $\mathrm{Sgr} \mathrm{A}^{*}$. If S0-2 started out as part of a binary on a very low specific energy (i.e., roughly parabolic) orbit with peribothron $\sim q$, then to become bound with $E_{b}$, it would have

\footnotetext{
${ }^{1}$ If the central region is populated by a cluster of stellar-mass black holes as argued by Miralda-Escudé \& Gould (2000), then the local mass density is a factor $\sim 7$ lower, and hence the relaxation time is a factor $\sim 7$ longer.
} 
to have been ejected from the binary with a velocity

$$
\Delta v=\frac{E_{b}}{v}=\sqrt{\frac{G M_{\mathrm{bh}}}{8 q}}(1-e) \sim 210 \mathrm{~km} \mathrm{~s}^{-1},
$$

where $v=\left(2 G M_{\mathrm{bh}} / q\right)^{1 / 2}$ is the velocity of the binary center of mass at peribothron. If we now equate $\Delta v$ with the internal velocity of $\mathrm{S} 0-2$ relative to the binary's center of mass,

$$
\Delta v=\sqrt{\frac{G m_{\mathrm{tot}}}{a_{\mathrm{bin}}}} \frac{m_{2}}{m_{\mathrm{tot}}},
$$

where $m_{1}$ and $m_{2}$ are the masses of S0-2 and its former companion, $m_{\mathrm{tot}}=m_{1}+m_{2}$, and $a_{\mathrm{bin}}$ is the binary's semimajor axis, we obtain

$$
a_{\mathrm{bin}}=q \frac{8}{(1-e)^{2}} \frac{m_{2}^{2}}{M_{\mathrm{bh}} m_{\mathrm{tot}}} .
$$

Finally, we assume that the binary can be broken apart tidally when the accelerations are related by

$$
\frac{2 G M_{\mathrm{bh}} a_{\mathrm{bin}}}{q^{3}}=\eta \frac{G m_{\mathrm{tot}}}{a_{\mathrm{bin}}^{2}}
$$

where $\eta \sim 1$ is a parameter. We then find

$$
\frac{m_{2}^{3}}{m_{\mathrm{tot}}^{2}}=\frac{\sqrt{\eta}}{32}(1-e)^{3} M_{\mathrm{bh}} \sim 200 \sqrt{\eta} M_{\odot},
$$

and

$$
a_{\mathrm{bin}} \frac{m_{2}}{m_{\mathrm{tot}}}=\frac{\sqrt{\eta}}{4}(1-e) q \sim 4 \sqrt{\eta} \mathrm{AU} .
$$

Equation (6) allows us to estimate the mass of the primary star and equation (7) the binary's semimajor axis. Note that in the limit, $m_{2} \gg m_{1}$, the left-hand sides of equations (6) and (7) become $m_{2}$ and $a_{\text {bin }}$, respectively. On the other hand, for a roughly equal mass ratio, $m_{1} \sim m_{2} \sim 800 \eta^{1 / 2} M_{\odot}$. Such a massive S0-2 would be much brighter than is observed. Hence, we will generally work in the regime $m_{2} \gg m_{1}$.

Equation (6) implies that the former companion of S0-2 was quite massive, with mass of order several hundred solar masses. However, since this equation contains the parameter $\eta$, which depends on the specific internal orbital parameters of the binary, numerical simulations are required to determine the actual range of plausible masses for the companion.

\section{NUMERICAL ESTIMATES}

\subsection{Single Encounters}

We conduct a series of simulations, in each of which the masses $m_{1}$ and $m_{2}$ are held fixed, but all of the other binary parameters are allowed to vary. The three binary orbital orientation angles and the orbital phase are randomly chosen. The binary eccentricity is drawn uniformly in $e_{\text {bin }}^{2}$ (but capped $e_{\text {bin }}<0.95$ ), and the semimajor axis is drawn uniformly in $\log a$ for $1 \mathrm{AU}<a<10 \mathrm{AU}$. We find that encounters leading to secondary orbits with the properties of S0-2's orbit, $q=125 \mathrm{AU}$ and $e=0.87$, have initial peribothra that are about 5 AU larger, i.e., $q_{\text {init }} \sim 130$ AU. All simulated encounters therefore begin with the binary on a parabolic orbit with $q_{\text {init }}$ at this value. Each set of simulations contains 20,000 integrations of the entire encounter between the massive-star binary and the massive black hole.

For each set of simulations (determined by choice of $m_{1}$ and $m_{2}$ ) we examine the cumulative distributions of three binary input parameters $\left(a_{\mathrm{bin}}, e_{\mathrm{bin}}\right.$, and $\left.\cos i_{\mathrm{bin}}\right)$ for the subset of encounters that lead to an S0-2-like orbit, namely, $0.86 \leq e<0.88$. We also examine the final peribothra $q$ of these orbits to ensure that they are consistent with the observed value for S0-2. Figure 1 shows the results for the 137 encounters meeting this condition for the simulation with $m_{1}=15 M_{\odot}$ and $m_{2}=150 M_{\odot}$. Results for other mass combinations look similar.

The first point to note from Figure 1 is that less than 1\% of the 20,000 simulated encounters lead to S0-2-type orbits. Of course, this is partly because we have defined the acceptable eccentricity range extremely narrowly. However, many binaries simply do not break up, or if they do, they leave S0-2 in a marginally bound or unbound orbit. We return to this issue in $\S 3.2$.

Of the parabolic encounters that do yield S0-2-like orbits, the distribution of binary eccentricities is consistent with being uniform in $e_{\text {bin }}^{2}$, which is the same as the parent distribution of all encounters. Hence, the probability of producing an S0-2-like orbit does not strongly depend on $e_{\text {bin. }}$. By contrast, this probability depends strongly on the binary's inclination $i$, with prograde orbits $\left(\cos i_{\text {bin }} \sim 1\right)$ being much preferred over retrograde orbits $\left(\cos i_{\text {bin }} \sim-1\right)$. This is plausible, since it is easier to disrupt a prograde than retrograde binary.

As predicted by equation (7), successful encounters have $a_{\text {bin }} \sim 4$ AU. There are essentially no successes for $a_{\text {bin }}<2$ AU. The decline in success toward higher semimajor axis seems to be less severe than the decline toward lower values. For this reason, we have conducted additional simulations with input distributions uniformly distributed of $1 \mathrm{AU}<a_{\mathrm{bin}}<100 \mathrm{AU}$. We find that only about $10 \%$ of successful encounters have $a_{\text {bin }}>10 \mathrm{AU}$, with the overwhelming majority of these having $a_{\text {bin }}<30 \mathrm{AU}$.

Finally, we find that the distribution of final peribothra is sharply peaked near $q \sim 124 \mathrm{AU}$. This shows that it is unnecessary to simulate a broad range of $q_{\text {init }}$ because only those binaries with $q_{\text {init }} \sim 130 \mathrm{AU}$ have the potential to yield S0-2-like orbits.

Figure 2 shows the number of successful encounters (again defined by $0.86 \leq e<0.88$ ) as a function of the "mass function" $m_{f} \equiv m_{2}^{3} / m_{\text {tot }}^{2}$ for 15 simulations with $m_{1} / M_{\odot}=7.5,15$, and 30 , and $m_{2} / M_{\odot}=50,75,100,150$, and 200 . Note that, in conformity with equation (6), the number of successful encounters is approximately a function of $m_{f}$ rather than of the two component masses separately. Also in conformity with equation (6) is the fact that this function peaks at $m_{f} \sim 200 M_{\odot}$. There are essentially no successful encounters for $m_{f} \lesssim 40 M_{\odot}$, which, for $m_{1} \sim 15 M_{\odot}$, corresponds to a minimum mass for the former companion of $m_{2} \gtrsim 60 M_{\odot}$.

As mentioned above, the absolute rate of successful encounters is quite small. Part of the problem is our extremely narrow definition of "success": of course the a priori probability of exactly reproducing the a posteriori known orbit of S0-2 is vanishingly small. One might plausibly argue that if S0-2 had any orbit with $q=125 \mathrm{AU}$ and $e<0.9$ it might well have been measured. Such broadened acceptance would increase the number of "successes" by 

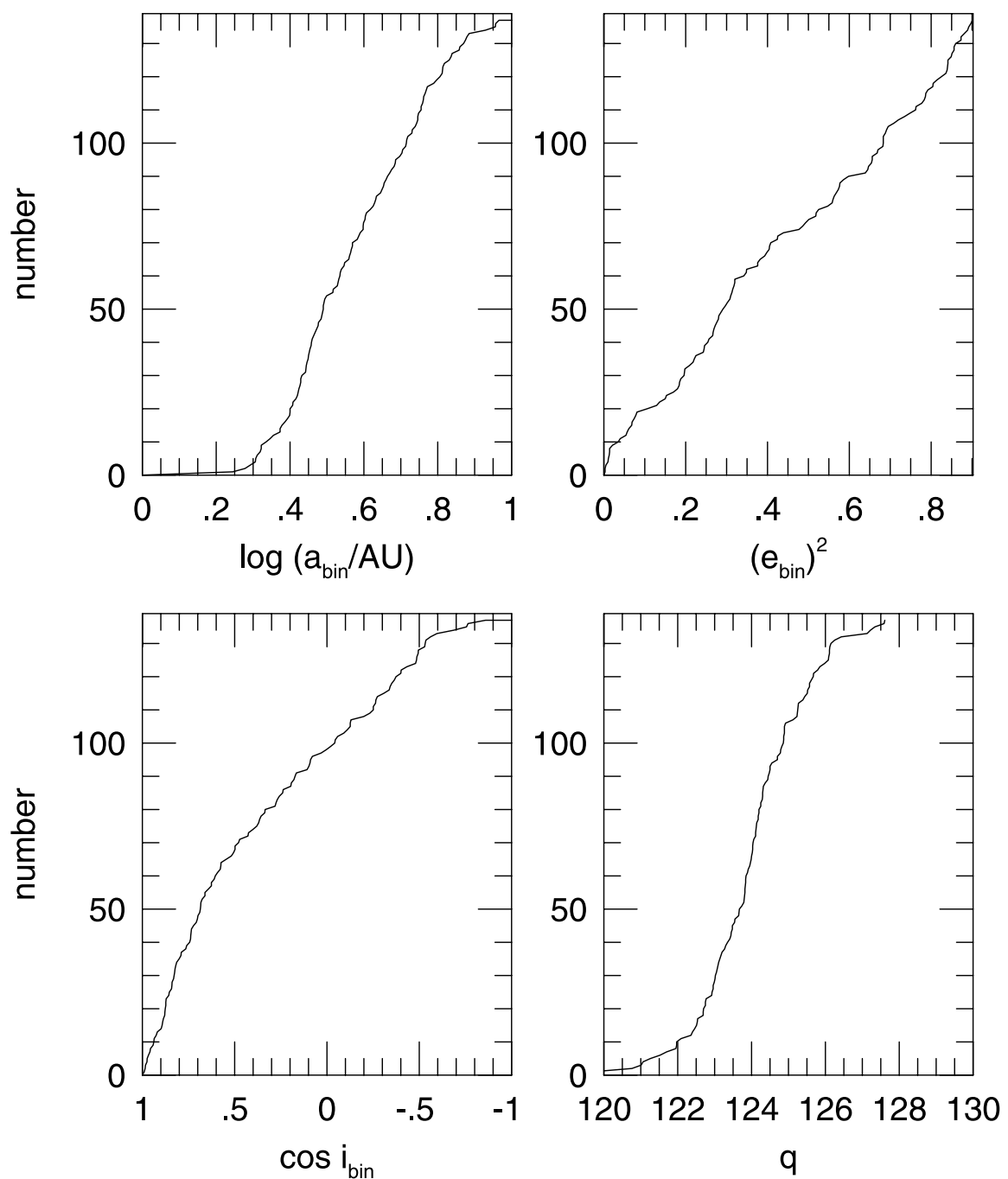

FIG. 1.-Cumulative distributions for "successful" tidal encounters between a binary with masses $\left(m_{1}, m_{2}\right)=\left(15 M_{\odot}, 150 M_{\odot}\right)$ and Sgr A*, with "success" defined as $m_{1}$ being injected into an orbit with eccentricity $0.86 \leq e<0.88$ (similar to S0-2). Shown are the semimajor axis $a_{\text {bin }}$, the eccentricity $e_{\text {bin }}$, and the inclination $i_{\text {bin }}$ of the incoming binary, as well as the peribothron $q$ of the final $m_{1}$ orbit. The simulated encounters were drawn uniformly in log $a_{\text {bin }}$, $e_{\text {bin }}^{2}$, and $\cos i_{\text {bin }}$. Hence "success" strongly favors prograde $\left(\cos i_{\text {bin }} \sim 1\right)$ orbits, and semimajor axes $a_{\text {bin }} \sim 4$ AU but does not depend strongly on $e_{\text {bin }}$. All simulated encounters had $q_{\text {init }}=130 \mathrm{AU}$, while the final peribothra are narrowly distributed around $125 \mathrm{AU}$.

about a factor 4 near the peak. The resulting fraction of successes is still fairly low.

\subsection{Repeated Encounters}

One should keep in mind, however, that the orbital parameters of the incident binary can be significantly changed even if it is not disrupted. As discussed in Appendix A, we expect that the incoming binary would not actually be on a parabolic orbit but rather on a highly elliptical one with a semimajor axis $a \sim 10^{4} \mathrm{AU}$, at which location a dense young cluster would be tidally disrupted. This induces only a slight $(\sim 5.4 \%)$ reduction in the energy requirements of the encounter. Within the formalism of $\S 2$, the effect is equivalent to decreasing $(1-e)$ by $5.4 \%$, or increasing $e$ from 0.87 to 0.877 . This reduces the overall mass scale for $m_{f}=m_{2}^{3} / m_{\text {tot }}^{2}$ by a factor $(1-0.054)^{3} \sim 0.85$ (see eq. [6]). Given the high mass scale of this equation and of Figure 2, this reduction is welcome, but modest.

However, this finite semimajor axis has another important effect: the binary reencounters $\mathrm{Sgr} \mathrm{A}^{*}$ on timescales of $\sim 10^{3} \mathrm{yr}$. When it does so, it preserves all the binary parameters it acquired in the last encounter, save the internal orbital phase relative to the phase of the orbit around Sgr A*, which is effectively randomized. Hence, thousands of such encounters may take place, which increase the chance that the binary will be disrupted. Partly, the internal binary orbit changes each time. However, even if the orbit is left unchanged, the mere randomizing of the internal orbital phase permits a new opportunity for a disruptive encounter.

To explore this effect, we simulate repeat encounters by binaries in orbit around $\mathrm{Sgr} \mathrm{A}^{*}$, continuing the simulation until the binary is disrupted or until it completes 1000 orbits. In order to permit efficient computation, we place the binaries in orbits with semimajor axis $a=3000 \mathrm{AU}$. Then, in order to be able to compare directly with the previous simulations we consider as a success, encounters with the same energy change, that is, with final orbits having eccentricity $e=0.847$. Finally, to minimize the role of Poisson fluctuations, we focus on the relative number of successes from the entire (up to 1000-orbit) simulation with the 


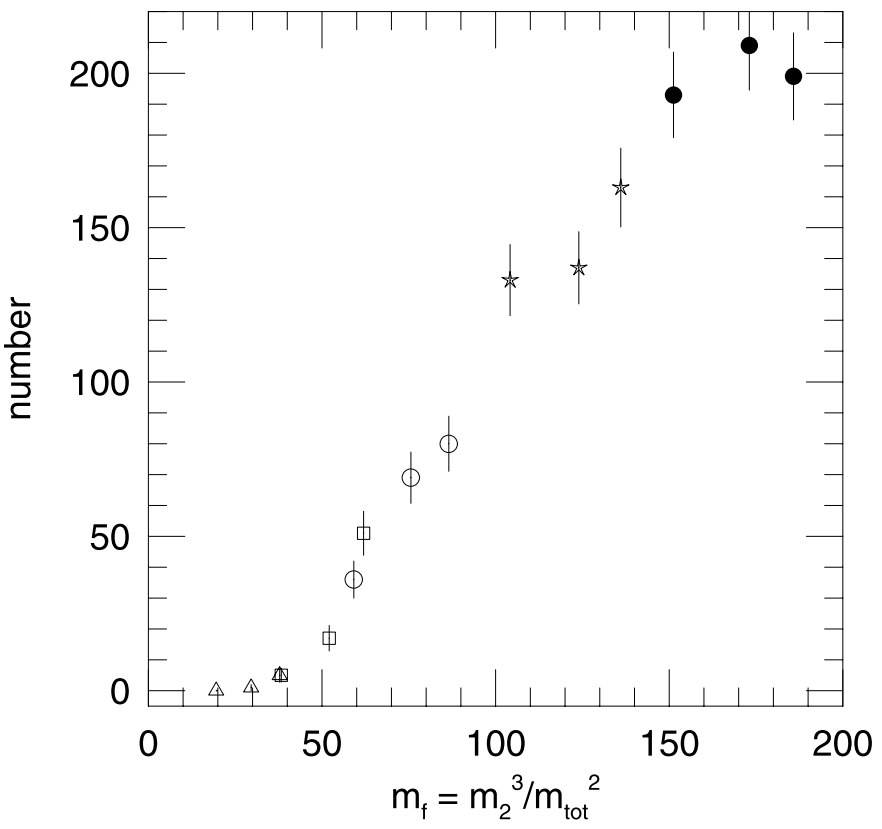

Fig. 2.-Number of "successful" tidal encounters (out of 20,000) between a binary with masses $\left(m_{1}, m_{2}\right)$ and Sgr A*, with " success" defined as $m_{1}$ being injected into an orbit with eccentricity $0.86<e<0.88$ (similar to $\mathrm{S} 0-2$ ). One may infer from eq. (6) that this success rate should be a function only of the combination $m_{f} \equiv m_{2}^{3} / m_{\text {tot }}^{2}$ with $m_{\text {tot }} \equiv m_{1}+m_{2}$. The figure bears out this conjecture. The red triangles, yellow squares, green circles, blue stars, and black solid circles refer, respectively, to $m_{2}=50,75$, 100,150 , and $200 M_{\odot}$. In each case, trials were run for $m_{1}=7.5,15$, and 30 $M_{\odot}$. The success rate peaks near $m_{f} \sim 200 M_{\odot}$, also in agreement with the naive prediction of eq. (6). [See the electronic edition of the Journal for a color version of this figure.]

number coming from the first encounter. For $m_{1}=15 M_{\odot}$ and $m_{2}=75 M_{\odot}$, the 1000 -orbit simulations double the number of successes, while for $m_{2}=100 M_{\odot}$ the improvement rises to about a factor 2.5.

About $90 \%$ of the post-first-orbit successes occur within the first 100 orbits, and none past 200 orbits, indicating that our 1000-orbit simulations were quite adequate. The great majority of additional successes have initial semimajor axes $1 \mathrm{AU}<a_{\text {bin }}<2.5 \mathrm{AU}$. Of those that do not, all but one are in retrograde orbits. That is, the additional successes arise because binaries with unfavorable parameters are gradually perturbed into a more favorable regime. Given the enhanced probability for binaries with $a_{\text {bin }}$ in the lowest range simulated, we conduct an additional simulation with $m_{2}=100 M_{\odot}$ and $0.1 \mathrm{AU}<a_{\text {bin }}<1 \mathrm{AU}$. However, we find very few successful encounters from this range.

In brief, allowing for multiple encounters in repeated orbits roughly doubles the number of successful encounters, i.e., those leaving the secondary in an orbit similar to $\mathrm{S} 0-2$.

\section{DISCUSSION}

S0-2 is the only close companion of Sgr A* with welldetermined orbital parameters and a high-quality spectroscopic identification. However, there could be many other remnants of disrupted massive-star binaries still waiting to be discovered. We found from our numerical integrations that binaries were more often disrupted leaving a star in a bound orbit with an eccentricity exceeding that of $\mathrm{S} 0-2$. We argued in $\S 3$ that the binary from which S0-2 was ejected had a mass function of at least $m_{f} \equiv m_{2}^{3} / m_{\text {tot }}^{2} \sim 40 M_{\odot}$. However, from equation (6), binaries of this $m_{f}$ should typically eject stars into orbits with eccentricity $e \sim 0.92$. For orbits with the same $q=125 \mathrm{AU}$ as S0-2, this would imply a period longer by a factor $[(1-0.87) /(1-$ $0.924)]^{3 / 2} \sim 2.2$, i.e., more than 30 yr. While such a star may have already been identified in near-infrared images, it would not yet be known whether the star would experience close approaches to $\mathrm{Sgr} \mathrm{A}^{*}$. For example, the stars $\mathrm{S} 0-16$ and $\mathrm{S} 0-19$ appear to have had close passages to Sgr A*, but their orbits are not yet well constrained (Ghez et al. 2003b). Similarly, stars with the same eccentricity as S0-2 but having larger peribothra would also have periods too long for one to have yet determined their orbits. Hence, there may well be a substantial population of highly eccentric young stars orbiting $\mathrm{Sgr} \mathrm{A}^{*}$ that were injected into their present orbits by the disruption of massive-star binaries. If so, these remnants could be used to probe statistically the recent formation of very massive stars in the Galactocentric region, even though the massive stars themselves have long since perished.

We thank Jordi Miralda-Escudé and Dan Watson for stimulating discussions and Angelle Tanner for useful comments on the manuscript. Work by A. G. was supported by grant AST 02-01266 from the NSF.

\section{APPENDIX A}

\section{FEEDING DELICACIES TO THE MONSTER}

What mechanism might feed a substantial number of massive-star binaries to orbits with peribothra $q_{\text {init }} \lesssim 130$ AU from which Sgr A* can "grab" an S0-2-like star? From $\S 3$, only $\sim 5 \%$ of binaries with semimajor axes in the decade $1 \mathrm{AU}<a_{\text {bin }}<10 \mathrm{AU}$ are captured in this manner. Moreover, only a small fraction, perhaps $O(10 \%)$, of massive stars will have a companion that is several times lighter orbiting with $a_{\mathrm{bin}}$ in this range. Hence, perhaps only $\sim 0.5 \%$ of massive stars passing within $q \sim 130$ AU will inject lighter companions into S0-2-like orbits. Since massive stars are fairly rare and the phase space permitted for S0-2 progenitors is quite small, it would seem necessary that these progenitors be preferentially funneled into this phase space if the encounters discussed in $\S \S 2$ and 3 are to plausibly account for the existence of S0-2.

Gerhard (2001) suggested that the He I stars in the central parsec of the Galactic center were born in a $10^{4}-10^{6} M_{\odot}$ cluster that was formed more than $30 \mathrm{pc}$ away from Sgr A* (like the Arches and Quintuplet clusters). The cluster sank inward to Sgr A* as a result of dynamical friction and was tidally disrupted by the massive black hole, thus leaving massive stars within the central parsec. Consider a cluster of mass $M_{\mathrm{cl}}$ and velocity dispersion $\sigma_{\mathrm{cl}}$ that has been formed outside the central parsec of the Galactic center. By the virial theorem, it will have an effective radius $R_{\mathrm{cl}} \sim G M_{\mathrm{cl}} / \sigma_{\mathrm{cl}}^{2}$. The cluster will sink by dynamical 
friction until it is tidally ripped apart at a separation from $\operatorname{Sgr} \mathrm{A}^{*}, r_{d}$,

$$
r_{d} \sim R_{\mathrm{cl}}\left(\frac{M_{\mathrm{bh}}}{M_{\mathrm{cl}}}\right)^{1 / 3} \sim \frac{G M_{\mathrm{bh}}^{1 / 3} M_{\mathrm{cl}}^{2 / 3}}{\sigma_{\mathrm{cl}}^{2}}=1.7 \times 10^{4} \mathrm{AU}\left(\frac{M_{\mathrm{cl}}}{10^{4} M_{\odot}}\right)^{2 / 3}\left(\frac{\sigma_{\mathrm{cl}}}{60 \mathrm{~km} \mathrm{~s}^{-1}}\right)^{-2} .
$$

For a star from this disrupted cluster to come within $q_{\text {init }}$ of $\operatorname{Sgr} \mathrm{A}^{*}$, it must have angular momentum $J<\left(2 G M_{\text {bh }} q_{\text {init }}\right)^{1 / 2}$ and therefore transverse velocity $v_{\perp}<\left(2 G M_{\mathrm{bh}} q_{\text {init }}\right)^{1 / 2} / r_{d}$. Hence,

$$
\frac{v_{\perp}}{v_{\mathrm{circ}}} \sim \frac{2 \sigma_{\mathrm{cl}}}{v_{q}}\left(\frac{M_{\mathrm{bh}}}{M_{\mathrm{cl}}}\right)^{1 / 3}=0.12\left(\frac{M_{\mathrm{cl}}}{10^{4} M_{\odot}}\right)^{-1 / 3} \frac{\sigma_{\mathrm{cl}}}{60 \mathrm{~km} \mathrm{~s}^{-1}}
$$

where $v_{\text {circ }}=\left(G M / r_{d}\right)^{1 / 2}$ is the circular speed at breakup and $v_{q}=6450 \mathrm{~km} \mathrm{~s}^{-1}$ is the velocity at peribothron. Hence, if the cluster is on a roughly circular orbit when it is disrupted, then essentially none of its stars will have a close passage to Sgr A*.

On the other hand, if the cluster is on a roughly radial orbit, then a fraction $f \sim 1-\exp \left[\left(-q v_{q} / r_{d} \sigma_{\mathrm{cl}}\right)^{2} / 2\right]$ will have close passages. That is,

$$
f \sim-\ln (1-f) \sim 0.35\left(\frac{M_{\mathrm{cl}}}{10^{4} M_{\odot}}\right)^{-4 / 3}\left(\frac{\sigma_{\mathrm{cl}}}{60 \mathrm{~km} \mathrm{~s}^{-1}}\right)^{2},
$$

so that for parameters that are plausible for a cluster formed in the deep gravitational well of the Galactic center, a substantial fraction of its members could come within $q_{\text {init }} \sim 130$ AU following a radial-orbit disruption. Clusters like the Arches and Quintuplet that are formed in the dense environment of the Galactic center could well have a high Jeans mass and therefore be dominated by very massive stars. In the above example, a massive-star cluster with $M_{\mathrm{cl}} \sim 10^{4} M_{\odot}$ would then contain of order $10^{2} 100 M_{\odot}$ stars. Of these one-third would come within $q=130 \mathrm{AU}$ of Sgr A* and, as estimated above, of order $0.5 \%$ of these might kick out companions onto S0-2-like orbits. Over the lifetime of S0-2, several such clusters might form and disrupt. Assuming that these clusters were on radial orbits, they are a plausible source of S0-2-like stars.

The final question then is whether it is plausible that such clusters will find themselves on radial orbits. To address this question, we first show that for a power-law density profile $\rho \propto r^{-\nu}$ in a background Kepler potential, dynamical friction will tend to circularize orbits for $\nu>3 / 2$ and make them more eccentric for $\nu<3 / 2$. Since the maximum bound velocity in a Kepler potential is $v_{\max }(r)=\left(2 G M_{\mathrm{bh}} / r\right)^{1 / 2}$, the amount of available phase-space scales as $\left[v_{\max }(r)\right]^{3} \propto r^{-3 / 2}$. Hence, for $\nu=3 / 2$, the phase-space density $f(u, r)$ is a constant, $f_{0}$, throughout the potential. The Chandrasekhar formula for the drag acceleration on a particle of mass $m$ and velocity $\boldsymbol{v}$ is then $\boldsymbol{a}=-k \boldsymbol{v}$, where

$$
k=\frac{4 \pi G^{2} m_{\mathrm{amb}} m}{v^{3}} \int_{0}^{v} d u 4 \pi u^{2} f(u, r) \ln \Lambda=\frac{16 \pi^{2} G^{2} m_{\mathrm{amb}} m f_{0}}{3} \ln \Lambda,
$$

and where $m_{\mathrm{amb}}$ is the mean mass of the ambient particles. Since $\ln \Lambda$ depends only very weakly on radius, $k$ is essentially independent of radius.

Over the course of a single orbital period $P$, an object with specific energy $E$ and specific angular momentum $\boldsymbol{L}$ will suffer mean rates of energy and angular momentum loss of

$$
\left\langle\frac{d E}{d t}\right\rangle=\frac{\oint d t \boldsymbol{v} \cdot \boldsymbol{a}}{P}=-\frac{k}{P} \oint d t v^{2}=2 k E
$$

and

$$
\left\langle\frac{d \boldsymbol{L}}{d t}\right\rangle=\frac{\oint d t \boldsymbol{r} \times \boldsymbol{a}}{P}=-\frac{k}{P} \oint d t \boldsymbol{L}=-k \boldsymbol{L} .
$$

Since $1-e^{2}=-2 L^{2} E /\left(G M_{\mathrm{bh}}\right)^{2}$, the evolution of the eccentricity can be written

$$
\frac{d \ln \left(1-e^{2}\right)}{d t}=2 \frac{\langle d L / d t\rangle}{L}+\frac{\langle d E / d t\rangle}{E}=-2 k+2 k=0 .
$$

For steeper profiles, the phase-space density is higher than average at pericenter; hence, the additional drag there tends to circularize the orbit, while for shallower profiles the higher phase-space density at apocenter tends to make them more eccentric.

Over most radii, the stellar mass profile around $\mathrm{Sgr} \mathrm{A}^{*}$ is a power law with $\nu \sim 1.8$. Hence, there is a weak tendency to circularize orbits. If there were a core in the mass distribution of radius $\sim 3^{\prime \prime}$, corresponding to $\sim 2500$ AU, then this core region could drive clusters toward eccentric orbits. That is, once the peribothron fell below the core radius, the declining dynamical friction inside the core (relative to that due to a power law) would drive the orbits to greater eccentricity and so still lower peribothron.

However, from equation (A1), it is clear that this process could not continue very long before the cluster was disrupted, unless the cluster had rather extreme parameters. On the other hand, from equation (A2), the cluster orbit must be highly eccentric if a significant fractions of its disrupted contents is to reach $q_{\text {init }}$. Dynamical friction by itself probably cannot produce such orbits. If a cluster had the required orbit to deliver S0-2 progenitors to Sgr A*, it must have been born on it. This in itself is not implausible: it is certainly possible for clusters to form from colliding clouds of gas whose angular momenta roughly cancel. Whether this happens frequently or infrequently is today, however, a matter of speculation. 
Alexander, T. 1999, ApJ, 527, 835

REFERENCES

Eckart, A., \& Genzel, R. 1996, Nature, 383, 415

Eckart, A., Genzel, R., Ott, T., \& Schodel, R. 2002, MNRAS, 331, 917

Gerhard, O. 2001, ApJ, 546, L39

Ghez, A. M., Becklin, E., Duchene, G., Hornstein, S., Morris, M., Salim,

S., \& Tanner, A. 2003b, Astron. Nachr., 324, 3

Ghez, A. M., Klein, B. L., Morris, M., \& Becklin, E. E. 1998, ApJ, 509, 678

Ghez, A. M., Morris, M., Becklin, E. E., Tanner, A., \& Kremenek, T. 2000, Nature, 407, 349

Ghez, A. M., et al. 2003a, ApJ, 586, L127

Heggie, D. C., Hut, P., \& McMillan, S. L. W. 1996, ApJ, 467, 359

Miralda-Escudé., J., \& Gould, A. 2000, ApJ, 545, 847

Salim, S., \& Gould, A. 1999, ApJ, 523, 633

Schodel, R., et al. 2002, Nature, 419, 694 